\title{
ALS and Cancer
}

\author{
Masamitsu Yamaguchi ${ }^{1^{*}}$, Yumiko Azuma ${ }^{2}$ and Hideki Yoshida ${ }^{1}$ \\ ${ }^{1}$ Department of Applied Biology, The Center for Advanced Insect Research, Kyoto Institute of Technology, Matsugasaki, Sakyo-ku, Kyoto, 606-8585, Japan \\ ${ }^{2}$ Department of Neurology, Graduate School of Medical Science, Kyoto Prefectural University of Medicine, 465 Kajii-cho, Kamigyo-ku, Kyoto, 602-8566, Japan \\ *Corresponding author: Masamitsu Yamaguchi, Department of Applied Biology, Kyoto Institute of Technology, Matsugasaki, Sakyo-ku, Kyoto, 606-8585, Japan, E-mail: \\ myamaguc@kit.ac.jp
}

Received date: October 26, 2016; Accepted date: October 27, 2016; Published date: October 31, 2016

Copyright: (c) 2016 Yamaguchi M, et al. This is an open-access article distributed under the terms of the Creative Commons Attribution License, which permits unrestricted use, distribution, and reproduction in any medium, provided the original author and source are credited.

\section{Editorial}

Amyotrophic Lateral Sclerosis (ALS) is a rare but very severe disease. In ALS patients, defect in motor neuron accompanied with muscle atrophy progresses rapidly that results in defect in locomotion, swallowing and respiration. Patients normally die in 2 to 5 years after the onset of disease, if the artificial ventilator is not equipped. However, up to now there is no effective therapy. A number of epidemiological studies suggested that ALS relates to cancer. It has been shown that prostate cancer survivors are associated with a decreased risk of ALS [1]. In the contrary, a significantly elevated risk of ALS among survivors of melanoma and tongue cancer has been also reported [1]. ALS and cancer share defects in various cellular processes including cell survival, cell death and cell cycle. However, in ALS the defects results in progressive defect of motor neuron, while in cancer it results in uncontrolled cell survival and proliferation [1]. More than thirty ALS-causing genes have been identified and every year still new ALS-causing gene is reported. Some of them appear to be involved in not only ALS but also cancer as summarized below and in Table 1 .

\begin{tabular}{|c|c|c|}
\hline Gene & ALS & Cancer \\
\hline$V A P B$ & missense & fusion gene \\
\hline$U B Q L N$ & disruption (UBQLN2), missense & loss or under-expressed \\
\hline VCP & missense & elevated expression \\
\hline $\begin{array}{l}\text { EWSR } \\
1\end{array}$ & missense & translocation \\
\hline TAF15 & missense & fusion gene \\
\hline$T D P-43$ & missense & upregulated expression \\
\hline FUS & $\begin{array}{l}\text { missense, in-frame deletion, } \\
\text { frameshift }\end{array}$ & fusion gene \\
\hline $\begin{array}{l}\text { MALAT } \\
1\end{array}$ & binding to TDP-43 & highly expressed \\
\hline NEAT1 & binding to TDP-43 and FUS & overexpressed \\
\hline $\begin{array}{l}\text { C9orf7 } \\
2\end{array}$ & hexanucleotide repeat expansion & $\begin{array}{l}\text { hexanucleotide } \\
\text { expansion }\end{array}$ \\
\hline
\end{tabular}

Table 1: Summary of genes related to both ALS and cancer.

Vesicle associated membrane protein-associated protein B (VAPB) localizes to both endoplasmic reticulum (ER) and mitochondria, where it involves in the ER-associated unfolded protein response and calcium exchange between ER and mitochondria [2]. A missense mutation in the coding region of VAPB has been identified in ALS patients and mitochondrial dysfunction appears to be a major cause for VAPBassociated ALS [2]. In association with cancer, the fusion gene VAPBIKZF3 (IKAROS family zinc finger 3) has been identified in breast cancer cells and it is suggested to be important for their growth and survival [3].

Ubiquilin family proteins (UBQLN1, UBQLN2, UBQLN3, UBQLN4 and UBQLN) contain the ubiquilin-like domain and the ubiquitin-associated domain. Disruption of UBQLN function is linked to various neurodegenerative diseases, including ALS. In fact, mutations in UBQLN2 are reported to cause ALS [4], likely due to the defect in transport of polyubiquitinated proteins to the proteasome [5]. Both UBQLN1 and UBQLN2 regulate cell migration, invasion, reorganization of actin cytoskeleton and epithelial mesenchymal transition. The $U B Q L N 1$ gene is lost or under-expressed in a large percent of human cancer cell lines [6]. Moreover frequent mutations in all five Ubiquilin family genes have been identified in lung cancer [6].

Valosin-containing protein (VCP) is an AAA ATPase that plays multiple roles such as morphological alteration of nuclear and Golgi membranes, transcriptional regulation, membrane fusion, programmed cell death, protein transportation and protein degradation. VCP is linked to the pathogenesis of ALS and other neurodegenerative diseases [7]. In relation to cancer, it is reported that the elevated expression of VCP correlates to the progression, prognosis and metastatic potential of esophageal carcinoma, colorectal carcinoma and prostate cancer. VCP promotes the growth, invasion, and metastasis of colorectal cancer [8].

Ewing sarcoma breakpoint region 1 (EWSRI) carrying RNAbinding domain plays a critical role in human $\mathrm{B}$ cell development and meiosis. Several translocations involving EWSR1 gene have been reported in cancer, such as $\mathrm{t}(11 ; 22)(\mathrm{q} 24 ; \mathrm{q} 12)$ chromosome translocation in Ewing's sarcoma and peripheral neuroepithelioma in which RNA-binding domain of EWSR1 is replaced with the DNAbinding domain of the transcription factor FLI-1 [1]. The other fusion of EWSR1 with the transcription factor ATF-1 or ERG is reported in malignant melanoma and Ewing family of tumors, respectively [1]. Missense variants of the EWSR1 gene are also reported in relation to ALS. In these ALS-specific variants, EWSR1 protein mislocalizes in motor neuron [1].

TATA-binding protein-associated factor 15 (TAF15) controls cellular viability by regulating cell cycle and cell death-related genes through a pathway involving a subset of the microRNA (miRNA), miR-17 family [1]. The fusion of TAF15 and the orphan nuclear receptor $C H N$ gene was identified in extra skeletal myxoid chondrosarcoma [1]. Thereafter, fusion genes of TAF15 with the transcription factor $C I Z / N M P 4$ gene and the orphan nuclear receptor NR4A3 gene were identified in acute leukemia and skeletal myxoid 
Page 2 of 3

chondrosarcoma, respectively [1]. In relation to ALS, nonsynonymous missense variants of TAF15 are reported in ALS patients [1]. These variants cause formation of cytoplasmic aggregates when expressed in primary cultures of spinal cord neurons and TAF proteins are found to be mislocalized in motor neurons in ALS patients [1].

TAR DNA-binding protein-43 (TDP-43) containing two RNA recognition motifs is involved in regulating RNA metabolism including transcription, splicing, mRNA stability, mRNA transport and miRNA biogenesis [1]. TDP-43 is a nuclear protein, but it mislocalizes in the cytoplasm as a component of ubiquitinated, cytoplasmic granules in the neurons of ALS and FTLD (frontotemporal lobar degeneration) patients [2]. Nearly 50 mutations in the TDP-43 gene have been identified in these neurodegenerative diseases [2]. In relation to cancer, a variant in a genomic region near the TDP-43 gene is mapped to be a strong Ewing sarcoma susceptibility locus [1]. It is also reported that expression of TDP-43 is upregulated in hepatocellular carcinoma cells [1].

Fused in sarcoma/translocated in liposarcoma (FUS/TLS or FUS) contains a single RNA recognition motifs and is also thought to be involved in regulation of RNA metabolism including transcription, splicing, mRNA stability, mRNA transport and miRNA biogenesis [1] The FUS gene was first identified as a fusion gene with the DNADamage-Inducible Transcript 3 (DDIT3, CHOP or GADD153) in myxoid liposarcomas with the translocation $\mathrm{t}(12 ; 16)(\mathrm{q} 13 ; \mathrm{p} 11)$ [9] The overexpression of FUS inhibits growth of prostate cancer cells, while its knockdown increases cell proliferation [1]. Mutations in FUS have been identified in ALS and FTLD patients [1]. Similarly to TDP-43, FUS was detected in the neuronal cytoplasmic inclusions in spinal motor neurons of ALS and FTD patients [1].

Based on the studies with ALS-causing RNA binding proteins as described above, attentions are currently paid on miRNAs in relation to both ALS and cancer [10,11]. The miRNAs are evolutionally conserved class of small, regulatory non-coding RNAs that negatively regulate expressions of protein coding genes and non-coding transcripts. They play critical roles in tumor initiation, progression and metastasis [11]. Deregulation of miRNA is reported in several cancers with both oncogenic and anti-oncogenic roles [11]. In ALS, the specific miRNAs, particularly miR-9, miR-125b, miR-132, miR-143 and miR-574 are found to be under control of both TDP-43 and FUS [10] These miRNAs appear to be a different set of those involving in cancer. However, miR-155 that is involved in sustained angiogenesis, evading immune surveillance and tumor-promoting inflammation and genomic instability in relation to cancer is also reported to be upregulated in ALS $[10,11]$.

Some of the long non-coding RNAs (lncRNA) are found to be involved in both ALS and cancer. The lncRNA are a group of noncoding RNAs composed of longer than 200 nucleotides [12]. They exhibit a variety of functions, including chromatin remodeling, mRNA splicing and editing, and transcriptional regulation. The lncRNA plays a key role in various cellular processes, including cell growth, apoptosis and carcinogenesis.

Metastasis-associated lung adenocarcinoma transcript 1 (MALAT1) is a nuclear lncRNA composed of more than 8,000 nucleotides, regulating alternative splicing by modulating the level of active serine/ arginine splicing factors [12]. MALAT1 is highly expressed in pancreatic cancer and its expression closely correlate with clinical stage, tumor size, metastasis in pancreatic cancer patients [13]. It is reported that downregulation of MALAT1 inhibits tumor cell proliferation and decreases cell migration and invasion in vitro [13]. MALAT1 is also highly expressed in clear renal cell carcinoma tissues and renal cancer cells and correlates with cell proliferation, migration and invasion of these cancer cells [13]. Similarly high expression of MALAT1 is reported in multiple myeloma, promoting cancer progression [13]. MALAT1 is also reported to promote proliferation and metastasis of osteosarcoma cells by activating the phosphoinositide 3-kinase/Akt pathway [13]. Nuclear enriched abundant transcript 1 (NEAT1), an lncRNA essential for the formation of nuclear body paraspeckles, is overexpressed in prostate cancer [14]. NEAT1 promotes myeloid differentiation. It is inhibited in acute promyelocytic leukemia patients by promyelocytic leukemia/retinoic acid receptor a, implicating a role of NEAT1 in acute promyelocytic leukemia [13].

By using individual-nucleotide resolution UV-crosslinking and immunoprecipitation, TDP-43 binding sites are mapped on the human RNAs [14]. Interestingly, preferential binding of TDP-43 to MALAT1 and NEAT1 in the brains of FTD patients are reported [14]. Moreover, it is demonstrated that TDP-43 and FUS are enriched in paraspeckles and bound to NEAT1 directly in ALS spinal motor neurons [14].

It should also be noted that the ALS-causing gene, C9orf72 produces noncoding transcripts with hexanucleotide repeat expansion [15]. Both sense and antisense C9orf72 transcripts are elevated in the brains of ALS patients where they form nuclear RNA foci [15]. In relation to cancer, correlation of C9orf72 transcripts carrying hexanucleotide repeat expansion to a dysplastic gangliocytoma is also implicated [16].

About $75 \%$ of causative factors of human diseases are conserved in Drosophila melanogaster. Based on the accumulation of lots of knowledge in genetics and developmental biology, the Drosophila models have been playing important roles for the study of human diseases including ALS and cancer [2]. Genetic screen with Drosophila ALS models identified the genes and signaling pathways that genetically interacts with the ALS-causing genes. Histone deacetylase 6 (HDAC6) has been identified as a modifier of TDP-43-associated phenotype [2]. Reducing HDAC6 expression rescues motor neuron dysfunction observed in flies carrying the TDP-43 A315T mutant gene [2]. In association with cancer, HDAC6 is overexpressed in clear cell renal cell carcinoma and it increases invasiveness and motility in kidney epithelial cells through deacetylation of a-tubulin [17]. Inhibition of HDAC6 exerts anti-tumor and anti-inflammation effects [18].

The rhomboid- 1 and -3 genes were identified as genetic interactants with the Drosophila FUS, cabeza gene [19]. Cabeza negatively regulates these genes that are positive regulators of the mitogenactivated protein kinase (MAPK) signaling pathway. In mammals, links between perturbations of the MAPK signaling pathway and ALS are also reported [20]. It is well known that mutations of components of MAPK signaling pathways are found in various cancers [20]. Considering the successful use of Drosophila model to identify genetic interactants with the ALS-causing genes, more extensive genetic screen with various ALS model flies will allow us to identify more genes and signaling pathways that are commonly related to ALS. These common genes and pathways could be promising targets for therapy of ALS. However, considering the mutual link between ALS and cancer [21], cautions have to be taken, since therapy designed for ALS may increase risk of cancer and vice versa. 
Page 3 of 3

\section{References}

1. Campos-Melo D, Droppelmann CA, Volkening K, Strong MJ (2014) RNA-binding proteins as molecular links between cancer and neurodegeneration. Biogerontology 15: 587-610.

2. Casci I, Pandey UB (2015) A fruitful endeavor: modeling ALS in the fruit fly. Brain Res 1607: 47-74

3. Edgren H, Murumagi A, Kangaspeska S, Nicorici D, Hongisto V, et al (2011) Identification of fusion genes in breast cancer by paired-end RNAsequencing. Genome Biol 12: R6.

4. Deng HX, Chen W, Hong ST, Boycott KM, Gorrie GH, et al. (2011) Mutations in UBQLN2 cause dominant X-linked juvenile and adult-onset ALS and ALS/dementia. Nature 477: 211-215.

5. Chang L, Monteiro MJ (2015) Defective Proteasome Delivery of Polyubiquitinated Proteins by Ubiquilin-2 Proteins Containing ALS Mutations. PLoS One 10: e0130162.

6. Shah PP, Lockwood WW, Saurabh K, Kurlawala Z, Shannon SP, et al. (2015) Ubiquilin1 represses migration and epithelial-to-mesenchymal transition of human non-small cell lung cancer cells. Oncogene 34 1709-1717.

7. Johnson JO, Mandrioli J, Benatar M, Abramzon Y, Van Deerlin VM, et al. (2010) Exome sequencing reveals VCP mutations as a cause of familial ALS. Neuron 68: 857-864.

8. Fu Q, Jiang Y, Zhang D, Liu X, Guo J, et al. (2016) Valosin-containing protein (VCP) promotes the growth, invasion, and metastasis of colorectal cancer through activation of STAT3 signaling. Mol Cell Biochem 418: 189-198.

9. Crozat A, Aman P, Mandahl N, Ron D (1993) Fusion of CHOP to a novel RNA-binding protein in human myxoid liposarcoma. Nature 363 640-644.

10. Volonte C, Apolloni S, Parisi C (2015) MicroRNAs: newcomers into the ALS picture. CNS Neurol Disord Drug Targets 14: 194-207.
11. Shah MY, Ferrajoli A, Sood AK, Lopez-Berestein G, Calin GA (2016) microRNA Therapeutics in Cancer - An Emerging Concept. EBioMedicine 12: 34-42.

12. Liu D, Yu X, Wang S, Dai E, Jiang L, et al. (2016) The gain and loss of long noncoding RNA associated-competing endogenous RNAs in prostate cancer. Oncotarget .

13. Zhang R, Xia LQ, Lu WW, Zhang J, Zhu JS (2016) LncRNAs and cancer. Oncol Lett 12: 1233-1239.

14. Nishimoto Y, Nakagawa S, Hirose T, Okano HJ, Takao M, et al. (2013) The long non-coding RNA nuclear-enriched abundant transcript 1_2 induces paraspeckle formation in the motor neuron during the early phase of amyotrophic lateral sclerosis. Mol Brain 6: 31 .

15. Wan P, Su W, Zhuo Y (2016) The Role of Long Noncoding RNAs in Neurodegenerative Diseases. Mol Neurobiol.

16. Ferrari R, Kero M, Mok K, Paetau A, Tienari PJ, et al. (2014) Familial frontotemporal dementia associated with C9orf72 repeat expansion and dysplastic gangliocytoma. Neurobiol Aging 35: 444.

17. Ramakrishnan S, Ku S (2016) HDAC 1 and 6 modulate cell invasion and migration in clear cell renal cell carcinoma. BMC Cancer 16: 617.

18. Yu J, Ma M (2016) HDAC6 inhibition prevents TNF-Ît-induced caspase 3 activation in lung endothelial cell and maintains cell-cell junctions. Oncotarget.

19. Shimamura M, Kyotani A, Azuma Y, Yoshida H, Nguyen TB, et al. (2014) Genetic link between Cabeza, a Drosophila homologue of Fused in Sarcoma (FUS), and the EGFR signaling pathway. Exp Cell Res 326: 36-45.

20. Kim EK, Choi EJ (2010) Pathological roles of MAPK signaling pathways in human diseases. Biochim Biophys Acta 1802: 396-405.

21. Gibson SB, Abbott D, Farnham JM, Thai KK, McLean H, et al. (2016) Population-based risks for cancer in patients with ALS. Neurology 87: 289-294. 